\title{
Cell Coverage Analysis of a Low Altitude Aerial Base Station in Wind Perturbations
}

\author{
Navuday Sharma*, Vishal Sharma ${ }^{\dagger}$, Maurizio Magarini ${ }^{\ddagger}$, Haris Pervaiz $^{\S}$, \\ Muhammad Mahtab Alam* and Yannick Le Moullec* \\ ${ }^{*}$ Thomas Johann Seebeck Department of Electronics, Tallinn University of Technology, Tallinn, Estonia \\ ${ }^{\dagger}$ Department of Information Security Engineering, Soonchunhyang University, Asan, South Korea \\ ${ }_{\ddagger}^{\ddagger}$ Department of Electronics, Information and Bio-Engineering, Politecnico di Milano, Milan, Italy \\ ${ }^{\S}$ School of Computing and Communications, Lancaster University, United Kingdom \\ email: \{firstname.lastname\}@taltech.ee*, vishal_sharma2012@ hotmail.com $^{\dagger}$, \\ maurizio.magarini@polimi.it ${ }^{\ddagger}$, h.b.pervaiz@lancaster.ac.uk ${ }^{\S}$
}

\begin{abstract}
The use of Unmanned Aerial Vehicles (UAVs) as Aerial Base Station (ABSs) is emerging as an effective technique to provide high capacity wireless networks to ground users. In this paper, cell coverage of a low altitude UAV is investigated for supporting such networks. An analytical framework for cell coverage area of an ABS is provided for Suburban, Urban and Urban high rise environments using a solid angle approach including radio link propagation effects in air-to-ground channel obtained from ray tracing simulations. Here, we account for the change in Euler angles such as roll, pitch and yaw due to perturbations by wind gusts or intentional maneuvers which leads to an increase in the geometrical coverage area by approximately 40-50 \%, given same transmission power and antenna gain of the $\mathrm{ABS}$.

Index Terms-Unmanned Aerial Vehicle, Aerial Base Station, Cell Coverage Area, Closed-form expression, Wind Perturbations and Air-to-Ground Channel.
\end{abstract}

\section{INTRODUCTION}

The rapid increase and further requirement of high-speed mobile data traffic lead to the adoption of the idea to bring access nodes as near as possible to the ground mobile users to have a higher capacity. This realization can be made feasible by using Unmanned Aerial Vehicles (UAVs) as Aerial Base Stations (ABS) ${ }^{1}$ [1], [2]. These can be deployed in real time by the operator based on the increase in the requirement of the data traffic from the ground users [3]. An ABS can be used both for supporting terrestrial networks and in remote locations in the absence of the terrestrial network [4]. There are many studies on the advancement of UAV into $5 \mathrm{G}$ and beyond on several aspects such as UAV classifications, standardization, security, channel modeling, cellular coverage, energy consumption, trajectory planning and positioning etc. Surveys [5], [6] provide a detailed review on such issues with many other aspects. Further, there has also been extensive work on cellular coverage provided by the UAVs, which can be categorized mainly as:

- Single UAV on the basis of altitude: The work in [7] investigates on achievable information rate and coverage for an ABS, considering Air-to-Ground (A2G) channel

\footnotetext{
${ }^{1}$ The terms UAV and ABS are interchangeably used throughout the paper.
}

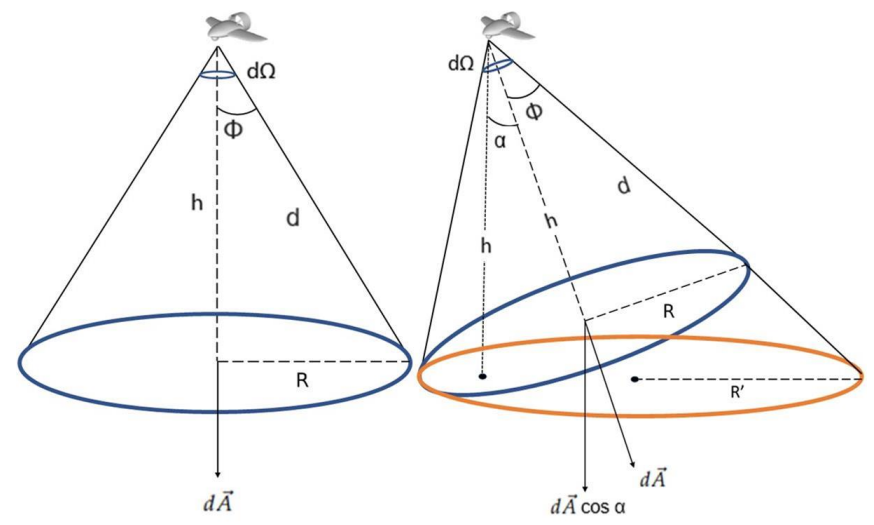

(a) Without rotation angle

(b) With rotation angle

Fig. 1. Coverage Area by ABS.

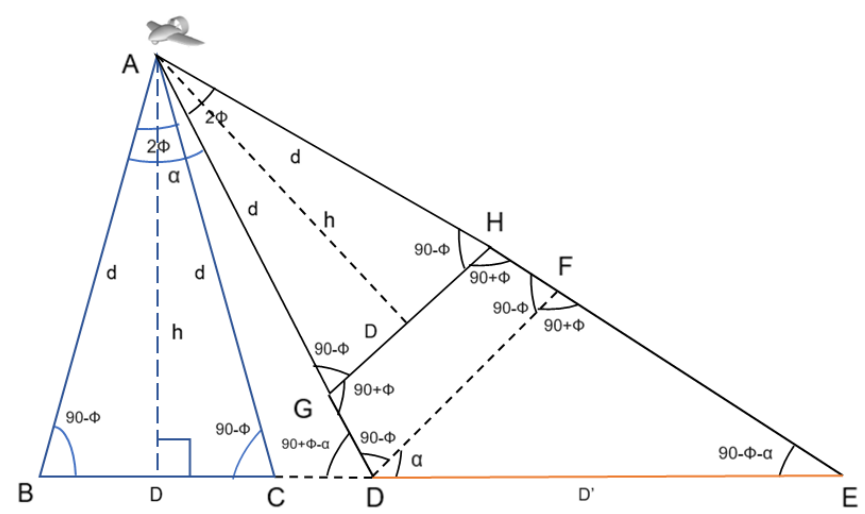

Fig. 2. Increase in Geometric Coverage Area.

with shadowing and random fading effects. In [8], coverage analysis for an ABS is performed using a ray tracing method of different altitudes and transmitted powers for Suburban, Urban and Urban High Rise scenarios developed from ITU-R specifications. The cell coverage and transmitting power results in [8] indicate the feasibility of using UAVs with Picocells and Fem- 
tocells formation with transmission power in the range of $33 \mathrm{dBm}$ and $20 \mathrm{dBm}$. Also, in [9], authors provide the mathematical model for optimal altitude of ABS for maximum coverage area and closed form equation for probabilistic line of sight (LoS) A2G model.

- Efficient placement of a UAV under specific constraints: References [10], [11] provide optimized placement of the UAVs for providing service to a maximum number of ground users with minimum transmit power. A similar study was performed in [12].

- Deployment of multiple UAVs: In [13], UAVs are deployed to improve network performance during occasional events with improvement in quality of coverage. Further, in [14] optimal coverage from UAVs is considered with minimizing their deployment delay and in [15], multiple ABS deployment using circle packing theory for maximum coverage is provided. Also, coverage performance of UAV assisted terrestrial network is presented in [16], where UAVs are cached, energy harvested, and deployed randomly in a 3D space. In [17], a stochastic approach is followed to obtain coverage probability for a ground user in the UAV network, taking into account the LoS probability, UAVuser distance and interference effects.

Also, in particular to the affect of wind perturbations on UAV movements, few articles emphasize on UAV control and coordination. References [18], [19] investigates on autonomous control of UAV swarms to avoid inter-UAV collision under wind perturbation using mean-field game theoretic control approach. To the best of our knowledge, this is the first paper addressing the novel closed-form expressions with proofs of cell coverage of an ABS, which do not exist currently. In this paper, a solid angle based approach is defined to describe the cell coverage area from an ABS analytically, which is not achievable via threshold received signal power approach [8]. Also, we introduce the rotation angle of ABS due to unavoidable wind gusts or intentional maneuvers and perform an analytical analysis to define the cell coverage equation with the information of critical parameters- altitude, elevation angle, rotation angle of $\mathrm{ABS}$, and propagation factors in A2G channel such as path loss, shadowing, and Rician fading, which are not available in the literature, bringing novelty to this work. Here, we implement the A2G channel model (and its parameters obtained from ray tracing) proposed in [8], [9], which significantly impacts the coverage performance at different ABS altitudes, as shown in numerical results. Also, we introduce the concept of increase of geometrical cell coverage due to rotation angle. Here, we assume the transmission by only an ABS and no terrestrial network. Also, modeling directivity function of ABS antenna for cellular coverage is out of the scope of this article.

The remaining of this paper is organized as follows. In Sec. II, A2G communication channel model is decribed. Sec. III and IV provide the analytical expressions for ABS, under different variable information available and expression for geometric increase in ABS coverage, respectively. Sec. V evaluates the performance of the expressions described in Sec. III and IV, followed by conclusions and future work in Sec. VI. Lastly, Appendix provides the proof of all analytical expressions.

\section{Air-TO-Ground Channel Model}

In this article, Close-In (CI) Reference Distance Path Loss model is implemented to obtain large scale fading characteristics such path loss (PL) and shadowing in A2G channel. Further, the inherent impact of probability of $\operatorname{LoS}$ is modeled via a closed form sigmoid approximated expression between the ABS and ground mobile user, as presented in [9]. Therefore, combining CI model and LoS probability, A2G channel is represented as follows:

$$
\begin{array}{r}
P L_{L o S}(d)[\mathrm{dB}]=20 \log _{10}\left(\frac{4 \pi d_{0}}{\lambda}\right)+10 n_{L o S} \log _{10}(d) \\
+X_{\sigma, L o S} \\
P L_{N L o S}(d)[\mathrm{dB}]=20 \log _{10}\left(\frac{4 \pi d_{0}}{\lambda}\right)+10 n_{N L o S} \log _{10}(d) \\
+X_{\sigma, N L o S}
\end{array}
$$

where, $P L$ is the path loss in $\mathrm{dB}, d$ is the radio link distance between ABS and ground user with $d_{0}=1 \mathrm{~m}$ as the reference distance, $\lambda$ is the wavelength, $n$ is the path loss exponent and $X_{\sigma}$ is the shadow fading. Also LoS (in subscript) implicates the parameters when the link is in line-of-sight condition and NLoS in non-LoS condition. In particular, the average path loss is given by

$$
\begin{array}{r}
P L(d)[\mathrm{dB}]=\mathbb{P}_{\mathrm{LoS}} \cdot P L_{L o S}(d)[\mathrm{dB}]+\left(1-\mathbb{P}_{\mathrm{LoS}}\right) . \\
P L_{N L o S}(d)[\mathrm{dB}],
\end{array}
$$

where, $\mathbb{P}_{\text {LoS }}$ represents the LoS probability which is modeled as a function of elevation angle between ABS and ground user, $\left(\theta_{i}\right)$ [20]:

$$
\mathbb{P}_{\text {LoS }}\left(\theta_{i}\right)=\frac{1}{1+a_{1} \exp \left\{-b_{1}\left(\theta_{i}-a_{1}\right)\right\}}
$$

Here, $a_{1}$ and $b_{1}$ are the environmental parameters obtained from building density and height from ITU-R specifications.

\section{AnAlyticAl FRAMEWORK FOR Drone CELL COVERAGE}

The coverage from an ABS is shown in Fig. 1a. For analytical simplifications, we assume an arbitrary circular area of radius $R$ with the cell coverage area by ABS defined using the solid angle. Solid angle is a $3 \mathrm{D}$ analogue of an angle, enclosed by a conical surface at the apex. Therefore, the solid angle $\Omega$ subtended by the circular cell area over the $\mathrm{ABS}$ is given by the expression

$$
\Omega=\int_{\theta_{1}}^{\theta_{2}} \int_{\phi_{1}}^{\phi_{2}} \sin \phi d \theta d \phi,
$$

where $d \phi$ is the elevation angle and $d \theta$ is the azimuth angle, with lower and upper limits as $\phi_{1}, \phi_{2}$ and $\theta_{1}, \theta_{2}$, respectively, subtended by the elemental area $d A$ on the ground at the 
ABS. Therefore, the expression of the cell coverage area can be given as

$$
\begin{aligned}
A=\frac{2 h^{2}}{R}\left(\sec \phi_{\max }-1\right) & \\
{\left[\left(\frac{1-\operatorname{erf}(a)}{2}\right)-\frac{1}{2} \exp \left(\frac{1-4 a b}{4 b^{2}}\right)\right.} & {\left[\operatorname{erf}\left(\frac{1}{2 b}-a\right)-1\right] . }
\end{aligned}
$$

where $h$ is the height of the ABS, $\phi_{\max }$ is the maximum elevation angle, depending on the type of antenna used, erf is the error function, $a$ and $b$ are substitution parameters, given in Appendix (A) along with the proof of (6). As such (6) does not consider the rotation angle of the ABS which can be introduced due to perturbations by wind gusts or maneuvers such as roll, pitch, and yaw. The expression for coverage area by the ABS including a certain rotation angle $\alpha$ is given as

$$
\begin{array}{r}
A=\frac{h^{2}}{R^{\prime}} \ln \left|\frac{1+\sin \alpha_{\max }}{1-\sin \alpha_{\max }}\right|\left(\sec \phi_{\max }-\sec \alpha_{\max }\right) \\
{\left[\left(\frac{1-\operatorname{erf}(a)}{2}\right)-\frac{1}{2} \exp \left(\frac{1-4 a b}{4 b^{2}}\right)\right.} \\
{\left[\operatorname{erf}\left(\frac{1}{2 b}-a\right)-1\right],}
\end{array}
$$

where $R^{\prime}$ is the radius of the geometrically increased coverage area as seen in Fig.1(b) and $\alpha_{\max }$ is the maximum rotation angle. The derivation of $R^{\prime}$ can be found in Appendix (D) and the proof of (7) is given in Appendix (B). This equation is only valid when $\alpha \neq n \pi$ for $n \epsilon[0,1,2,3, \ldots, \infty]$. For maintaining stable flight, $\alpha$ assumes a lower value and $\alpha \ngtr \frac{\pi}{2}$. From (7), the coverage area is known when the altitude and the rotation angle of the ABS is known. The coverage can also be obtained by the following expression when ABS altitude is unknown. The proof is available in Appendix (C).

$$
\begin{aligned}
& A=R^{\prime} \ln \left|\frac{1+\sin \alpha_{\max }}{1-\sin \alpha_{\max }}\right| \ln \left|\frac{\tan \frac{\phi_{\max }}{2}}{\tan \frac{\alpha_{\max }}{2}}\right| \\
& {\left[\frac{1}{6}-\frac{1}{2}\left[\operatorname{erf}(a)-\frac{1}{3} \exp \left(\frac{9}{4 b^{2}}-\frac{a}{b}\right)\right.\right.} \\
& {\left[\operatorname{erf}\left(\frac{-3}{2 b}+t\right)-1\right] . }
\end{aligned}
$$

For all the proofs, we have assumed $R, R^{\prime}$ and $h$ to be fixed. However, the cell coverage is a sub-area of the total circular area of radius $R$ and $R^{\prime}$. It depends on variables $\phi, \alpha$ and parameter $\mathbb{P}\left(P_{R X}(r) \geq P_{t h}\right)$, given in Appendix A.

\section{INCREASE IN THE GEOMETRIC COVERAGE AREA BY ABS}

Due to the rotation angle, the coverage area by the ABS is bound to increase geometrically, as shown in Fig. $1 \mathrm{~b}$. However, it also results in an increase in the A2G link propagation losses, such as path loss, shadowing and multipath effects with the same transmitting power and antenna gains.
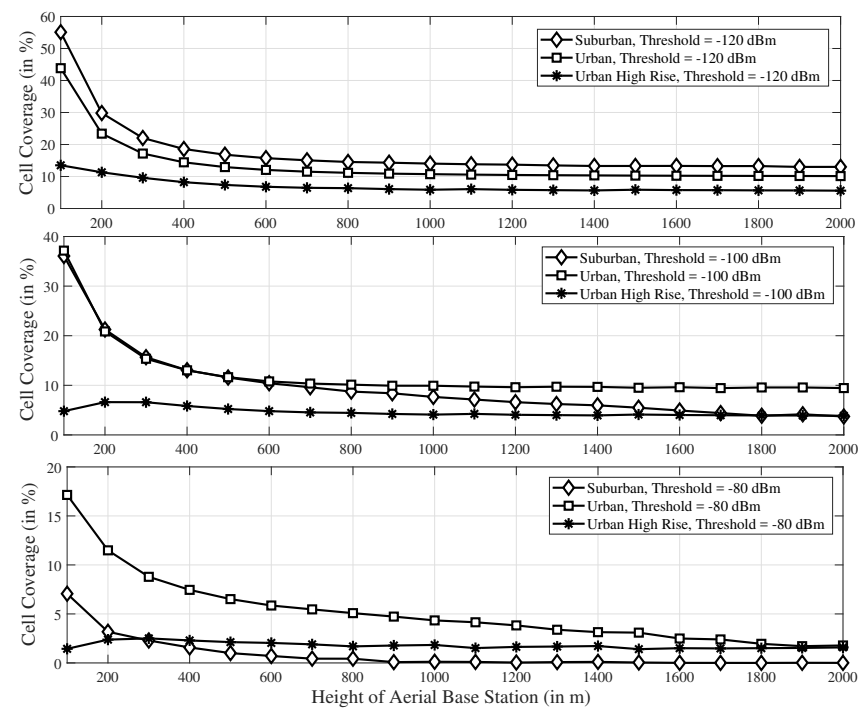

Fig. 3. Cell coverage with known ABS altitude without rotation.
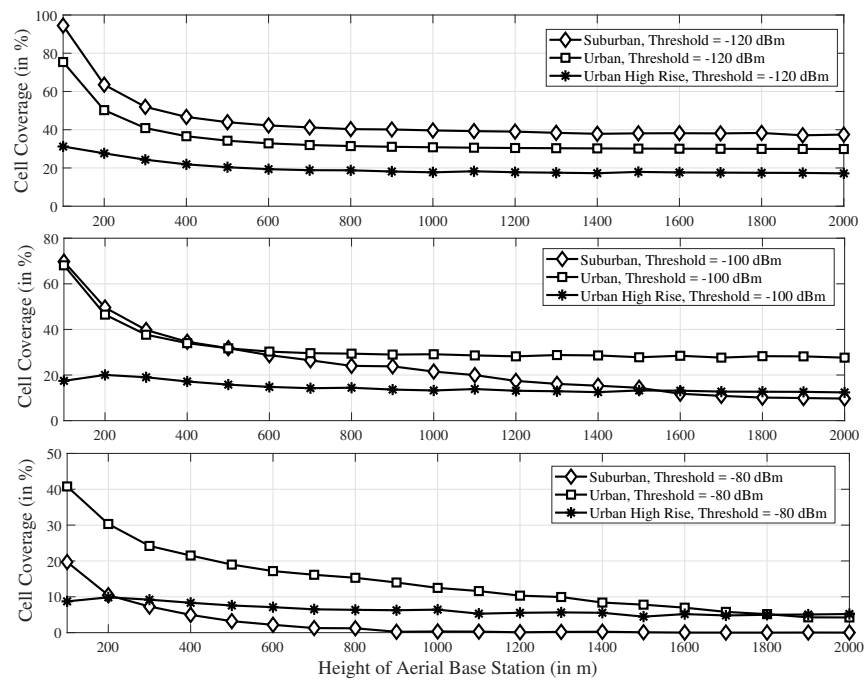

Fig. 4. Cell coverage with known ABS altitude with rotation.

Therefore, the final coverage area may increase or decrease depending on the propagation environment and effects. But, this geometric increase in coverage area needs to be taken into account into (7) and (8) to obtain the final coverage area. Fig. 2 shows a 2D layout of Fig. 1b for calculation of the radius of coverage area due to rotation. Thus, the radius $R^{\prime}$ of the coverage area due to rotation can be written as

$$
R^{\prime}=\frac{d \sin ^{2}(90+\phi) \sin 2 \phi}{2 \sin (90+\phi-\alpha) \sin (90-\phi) \sin (90-\phi-\alpha)} .
$$

In this section, we show the results at different ABS altitudes, for Suburban, Urban and Urban High Rise scenarios, with a constant transmission power of $18 \mathrm{dBm}$, from the previous analytical analysis. Fig. 3 corresponds to (6), while Fig. 4 corresponds to (7). Here, we use the ray tracing measurement results according to our previous work in [8], 
which includes all propagation link factors, for practical received signal strength indicator (RSSI) values from poor to good LTE connectivity, i.e, $-120,-100$ and $-80 \mathrm{dBm}$, respectively. From Fig. 3, the results are in correlation with [8], with higher cellular coverage at lower RSSI thus validating the feasibility of the closed-form expressions. The decrease in coverage for Suburban environments is much higher as compared to Urban due to changes in fading conditions experienced by the A2G channel, as detailed in [8]. Also, by introducing the rotation angle in the range of $\left[-20^{\circ}, 20^{\circ}\right]$, a significant increase in geometrical coverage can be observed from Fig. 4. However, by using non-directional antenna at ABS, the effect of rotation angle will be limited as compared to directional antenna.

\section{CONCLUSION AND FUTURE WORK}

This article provides the closed-form expressions with proofs for obtaining cellular coverage by an aerial base station, depending on the available variable information. Also, a geometric aspect of rotation angle due to wind perturbations or intentional maneuvers, is described. Finally, simulation results from realistic ray tracing measurements were used to verify the behavior of the graphs to define the feasibility of expressions. However, new measurements for link propagation losses with rotation angle and possible inclusion of multiple drone network will be part of our future activity.

\section{ACKNOWLEDGEMENT}

This project has received funding from the NATO-SPS funding grant agreement No. G5482 and the European Union's Horizon 2020 research and innovation program under grant agreement No. 668995 and is also partly supported by European Union Regional Development Fund in the framework of the Tallinn University of Technology Development Program 2016-2022. This material reflects only the authors view and the EC Research Executive Agency is not responsible for any use that may be made of the information it contains.

\section{APPENDIX}

\section{A. Proof for Coverage Area by ABS with known Altitude without Rotation Angle}

As seen from Fig. 1a, the solid angle subtended by an element on the ground at ABS is given by

$$
d \Omega=\frac{\overrightarrow{d A}}{d^{2}} \cdot \hat{n}
$$

where $d$ is the $3 D$ distance or the slant height of the cone formed from the geometry in Fig. 1 and $\hat{n}$ is the unit vector from the origin. The direction of $\hat{n}$ and $\overrightarrow{d A}$ is same when no rotation angle is present. From (10) we have

$$
d A=d^{2} d \Omega
$$

and from (5),

$$
d A=\sin \phi d^{2} d \theta d \phi
$$

This relationship is only based on the geometrical aspects of Fig. 1a. Therefore, the parameter $\mathbb{P}\left(P_{R X}(r) \geq P_{t h}\right)$ is added to the above expression. This produces the actual coverage by the ABS. This parameter denotes the probability that the received power by the ground user $\left(P_{R X}\right)$ is greater than the received power threshold $\left(P_{t h}\right)$ and $r$ is the $2 D$ distance between the ABS and the user. Thus we have

$$
d A=\sin \phi d^{2} \mathbb{P}\left(P_{R X}(r) \geq P_{t h}\right) d \theta d \phi d r .
$$

Thereby integrating (11), we have

$A=\frac{d^{2}}{A_{C}} \int_{\phi=0}^{\phi=\phi_{\max }} \sin \phi d \phi \int_{\theta=0}^{\theta=2 \pi} d \theta \int_{0}^{R} \mathbb{P}\left(P_{R X}(r) \geq P_{t h}\right) d r$,

where $R$ is the cell radius and corresponds to $\phi_{\max } . A_{C}=$ $\pi R^{2}$ is the cell area used to normalize the final cell coverage $A$. Thus, from Fig. 1 and (12) we have

$$
A=\frac{2 \pi h^{2}}{A_{C}} \int_{\phi=0}^{\phi=\phi_{\max }} \frac{\sin \phi}{\cos ^{2} \phi} d \phi \int_{0}^{R} \mathbb{P}\left(P_{R X}(r) \geq P_{t h}\right) d r .
$$

Now, integration of each part is shown separately as

$$
A=\frac{2 \pi h^{2}}{A_{C}} I_{1} I_{2}
$$

where

$$
I_{1}=\int_{\phi=0}^{\phi=\phi_{\max }} \frac{\sin \phi}{\cos ^{2} \phi} d \phi=\sec \phi_{\max }-1
$$

and

$$
I_{2}=\int_{0}^{R} \mathbb{P}\left(P_{R X}(r) \geq P_{t h}\right) d r .
$$

$\mathbb{P}\left(P_{R X}(r) \geq P_{t h}\right)$ can be calculated using Q-function as

$$
\mathbb{P}\left(P_{R X}(r) \geq P_{t h}\right)=Q\left(\frac{P_{t h}-\bar{P}_{R X}(r)}{\sigma}\right),
$$

where $\sigma$ is standard deviation of log-normal shadow fading. The Q-function is defined in as

$$
Q(x)=\int_{x}^{\infty} \frac{1}{\sqrt{2 \pi}} \exp \left(-\frac{y^{2}}{2}\right) d y=\frac{1}{2}\left[1-\operatorname{erf}\left(\frac{x}{\sqrt{2}}\right)\right],
$$

where $\operatorname{erf}(\cdot)$ is an error function. Therefore we have

$$
\mathbb{P}\left(P_{R X}(r) \geq P_{t h}\right)=\frac{1}{2}\left[1-\operatorname{erf}\left(\frac{P_{t h}-\bar{P}_{R X}(r)}{\sigma \sqrt{2}}\right)\right] .
$$

From the log-distance path loss model (in $\mathrm{dB}$ ),

$$
\bar{P}_{R X}(r)=P_{T X}+G_{T}+G_{R}-P L,
$$

and by substituting for $P L$, we have,

$$
\bar{P}_{R X}(r)=P_{T X}+G_{T}+G_{R}-\left(P L_{0}+10 \eta \log _{10}\left(\frac{r}{r_{0}}\right)\right),
$$

where $P_{T X}$ is the transmitted power, $G_{T}$ and $G_{R}$ are the transmitting and receiving antenna gain, respectively, PL is the path loss which is further written as a function of 
reference path loss $P L_{0}$, at distance $r_{0}$ from the transmitter, where and path loss exponent $\eta$. Therefore, we have

$$
\begin{array}{r}
\mathbb{P}\left(P_{R X}(r) \geq P_{t h}\right)=\frac{1}{2}[1-\operatorname{erf} \\
\left.\left(\frac{P_{t h}-\left[P_{T X}+G_{T}+G_{R}-\left[P L_{0}+10 \eta \log _{10}\left(\frac{r}{r_{0}}\right)\right]\right]}{\sigma \sqrt{2}}\right)\right],
\end{array}
$$

where we substitute the following for clarity

$$
\begin{gathered}
a=\left(\frac{P_{t h}-P_{T X}-G_{T}-G_{R}+P L_{0}+10 \eta \log _{10}\left(R / r_{0}\right)}{\sigma \sqrt{2}}\right) \\
\text { and } b=\frac{10 \eta \log _{10}(e)}{\sigma \sqrt{2}} .
\end{gathered}
$$

Therefore, we represent

$$
\begin{gathered}
\mathbb{P}\left(P_{R X}(r) \geq P_{t h}\right)=\frac{1}{2}-\frac{1}{2} \operatorname{erf}\left(a+b \ln \frac{r}{R}\right) \text { and } \\
I_{2}=\int_{0}^{R} \mathbb{P}\left(P_{R X}(r) \geq P_{t h}\right) d r= \\
\int_{0}^{R} \frac{1}{2}-\frac{1}{2} \operatorname{erf}\left(a+b \ln \frac{r}{R}\right) d r .
\end{gathered}
$$

Replacing $t=a+b \ln \frac{r}{R}$

$$
I_{2}=\frac{R}{2}-\frac{R}{2 b} \int_{-\infty}^{a} \exp \left(\frac{t-a}{b}\right) \operatorname{erf}(t) d t
$$

Integrating by parts, we obtain

$$
\begin{aligned}
I_{2}=R\left[\left(\frac{1-\operatorname{erf}(a)}{2}\right)-\frac{1}{2} \exp \right. & \left(\frac{1-4 a b}{4 b^{2}}\right) \\
& {\left[\operatorname{erf}\left(\frac{1}{2 b}-a\right)-1\right] . }
\end{aligned}
$$

Now substituting for $I_{1}, I_{2}$ and $A_{C}$ in (13), we finally get (6).

\section{B. Proof for Coverage area by ABS with known Altitude and} Rotation Angle

As seen from Fig. 1b, the coverage area of ABS tends to change due to the introduction of rotation angle $\alpha$. Therefore, (10), (11), (12) and (13) will be modified as

$$
\begin{gathered}
d \Omega=\frac{d A \cos \alpha}{d^{2}} \\
d A=\frac{1}{\cos \alpha} \sin \phi d^{2} \mathbb{P}\left(P_{R X}(r) \geq P_{t h}\right) d \theta d \phi d r d \alpha, \\
A=\frac{d^{2}}{A_{C}} \int_{\alpha=0}^{\alpha=\alpha_{\max }} \frac{d \alpha}{\cos \alpha} \int_{\phi=\alpha_{\max }}^{\phi=\phi_{\max }} \sin \phi d \phi \int_{\theta=0}^{\theta=2 \pi} d \theta \\
\int_{0}^{R} \mathbb{P}\left(P_{R X}(r) \geq P_{t h}\right) d r \\
A=\frac{2 \pi h^{2}}{A_{C}} I_{1} I_{2} I_{3},
\end{gathered}
$$

$$
I_{3}=\int_{\alpha=0}^{\alpha=\alpha_{\max }} \frac{d \alpha}{\cos \alpha}
$$

Thus far,

$$
I_{1}=\sec \phi_{\max }-\sec \alpha_{\max }
$$

$I_{2}$ remains unchanged and we obtain

$$
I_{3}=\frac{1}{2} \ln \left|\frac{1+\sin \alpha_{\max }}{1-\sin \alpha_{\max }}\right| .
$$

Substituting $I_{1}, I_{2}$ and $I_{3}$ in (17), we obtain (7).

\section{Proof for Coverage area by ABS with unknown Altitude and known Rotation Angle}

From Fig. 1, $d$ can be expressed as

$$
d=\frac{r}{\sin \phi} \text {. }
$$

Substituting in (15),

$$
d A=\frac{1}{\cos \alpha} \frac{r^{2}}{\sin \phi} \mathbb{P}\left(P_{R X}(r) \geq P_{t h}\right) d \theta d \phi d r d \alpha .
$$

By integrating, as done for (16), we have

$$
\begin{aligned}
A=\frac{1}{A_{C}} \int_{\alpha=0}^{\alpha=\alpha_{\max }} \frac{d \alpha}{\cos \alpha} \int_{\phi=\alpha_{\max }}^{\phi=\phi_{\max }} \frac{1}{\sin \phi} d \phi \int_{\theta=0}^{\theta=2 \pi} d \theta \\
\int_{0}^{R} r^{2} \mathbb{P}\left(P_{R X}(r) \geq P_{t h}\right) d r .
\end{aligned}
$$

Here $I_{1}$ and $I_{2}$ (from (13)) are updated as

$$
I_{1}=\int_{\phi=\alpha_{\max }}^{\phi=\phi_{\max }} \frac{1}{\sin \phi} d \phi=\ln \left|\frac{\tan \frac{\phi_{\max }}{2}}{\tan \frac{\alpha_{\max }}{2}}\right|,
$$

and

$$
\begin{aligned}
I_{2}=\int_{0}^{R^{\prime}} r^{2} \mathbb{P}\left(P_{R X}(r) \geq P_{t h}\right) d r= \\
\int_{0}^{R} r^{2}\left[\frac{1}{2}-\frac{1}{2} \operatorname{erf}\left(a+b \ln \frac{r}{R^{\prime}}\right)\right] d r .
\end{aligned}
$$

However $I_{3}$ remains the same. Following the same steps for solving $I_{2}$ by replacing $t=a+b \ln \frac{r}{R^{\prime}}$, we get

$$
\begin{aligned}
& \int_{0}^{R^{\prime}} r^{2} \mathbb{P}\left(P_{R X}(r) \geq P_{t h}\right) d r= \\
& \frac{R^{\prime 3}}{6}-\frac{R^{\prime 3}}{2 b} \int_{-\infty}^{a}\left[\exp \left(\frac{t-a}{b}\right)\right]^{3} \operatorname{erf}(t) d t,
\end{aligned}
$$

By integrating the above expression, we get

$$
\begin{aligned}
& \int_{0}^{R^{\prime}} r^{2} \mathbb{P}\left(P_{R X}(r) \geq P_{t h}\right) d r=\frac{R^{\prime 3}}{6}-\frac{R^{\prime 3}}{2} \\
& {\left[\operatorname{erf}(a)-\frac{1}{3} \exp \left(\frac{9}{4 b^{2}}-\frac{a}{b}\right)\left[\operatorname{erf}\left(\frac{-3}{2 b}+t\right)-1\right]\right] .}
\end{aligned}
$$

Substituting the above expressions in (18), the final expression obtained is (8). 


\section{Proof for increase in Geometric Coverage Area by ABS due to rotation angle}

From Fig. 2, $\triangle A B C$ is rotated by $\angle B A D=\alpha$ to form $\triangle A G H$. Therefore, the sides and angles of both triangles are equal. The elevation angle is $\angle \phi$ corresponding to Fig. 1 . Therefore, $\angle B A C$ and $\angle G A H$ are $2 \phi$. Also, corresponding to Fig. $1, A B=A C=A G=A H=d$ with height of both triangles equal to $h$. Without rotation, the diameter of covered area by the ABS is $B C=D$ and the diameter of covered area after rotation is $D E=D^{\prime}$. Here, we find the analytical expression for $D^{\prime}$ assuming that $D$ is known. From $\triangle A B C, \angle A B C=\angle A C B$ due to property of an isosceles triangle since $A B=A C$. As known from the property of a triangle, $\angle B A C+\angle A B C+\angle A C B=180^{\circ}$. This implies, $2 \phi+2 \angle A B C=180^{\circ}$. Therefore, $\angle A B C=$ $\angle A C B=90-\phi$. Similarly, from $\triangle A B D$, we have $\angle B A D+\angle A B D+\angle A D B=180^{\circ}$. This implies, $\alpha+90-$ $\phi \angle A D B=180^{\circ}$. Thus we have, $\angle A D B=90+\phi-\alpha$. Now, from the exterior angle property of the triangle, the measure of an exterior angle of a triangle is equal to the sum of the measures of its interior opposite angles. Thus, $\angle A D B=\angle D A E+\angle A E D$. This implies, $90+\phi-\alpha=$ $2 \phi+\angle A E D$ and $\angle A E D=90-\phi-\alpha$. Dropping a segment $D F$ on $A E$ such that $D F$ is parallel to $G H$. From Fig. 2, we observe $\angle A H G+\angle F H G=180^{\circ}$. Therefore, $\angle F H G=$ $90+\phi$. Following the same concept, $\angle D G H=90+\phi$. From the theory of corresponding angles, when two parallel lines are crossed by a transversal, its corresponding angles are equal. Therefore, $\angle A G H=\angle A D F=90-\phi$ and $\angle A H G=\angle A F D=90-\phi$. Also from geometry, $\angle A D B+$ $\angle A D F+\angle F D E=180^{\circ}$. This implies, $90+\phi-\alpha+90-$ $\phi+\angle F D E=180^{\circ}$. Therefore, $\angle F D E=\alpha$.

Now, from the Sine rule of triangles, in $\triangle A C D$

$$
\begin{gathered}
\frac{A C}{\sin \angle A D C}=\frac{A D}{\sin \angle A C D}=\frac{d}{\sin (90+\phi-\alpha)}=\frac{A D}{\sin (90+\phi)}, \\
A D=\frac{d \sin (90+\phi)}{\sin (90+\phi-\alpha)},
\end{gathered}
$$

Again from Sine rule in $\triangle A D F$

$$
\begin{gathered}
\frac{A D}{\sin \angle A F D}=\frac{D F}{\sin \angle D A F}, \\
\frac{d \sin (90+\phi)}{\sin (90+\phi-\alpha) \sin (90-\phi)}=\frac{D F}{\sin 2 \phi}, \\
D F=\frac{d \sin (90+\phi) \sin 2 \phi}{\sin (90+\phi-\alpha) \sin (90-\phi)},
\end{gathered}
$$

Now applying the Sine rule in $\triangle D E F$ yields

$$
\begin{aligned}
& \frac{D F}{\sin \angle D E F}=\frac{D E}{\sin \angle D F E}= \\
& \frac{d \sin (90+\phi) \sin 2 \phi}{\sin (90+\phi-\alpha) \sin (90-\phi) \sin (90-\phi-\alpha)}=\frac{D^{\prime}}{\sin (90+\phi)},
\end{aligned}
$$

Therefore, the radius of coverage area, i.e, half its diameter, is given in (9).

\section{REFERENCES}

[1] Y. Zeng, R. Zhang, and T. J. Lim, "Wireless communications with unmanned aerial vehicles: opportunities and challenges," in IEEE Communications Magazine, vol. 54, no. 5, pp. 36-42, May 2016.

[2] A. Fotouhi, M. Ding, and M. Hassan, "Flying Drone Base Stations for Macro Hotspots," in IEEE Access, vol. 6, pp. 19530-19539, 2018.

[3] N. Sharma, M. Magarini, D. N. K. Jayakody, V. Sharma, and J. Li, "On-Demand Ultra-Dense Cloud Drone Networks: Opportunities, Challenges and Benefits," in IEEE Communications Magazine, vol. 56, no. 8, pp. 85-91, August 2018.

[4] Y. Li and L. Cai, "UAV-Assisted Dynamic Coverage in a Heterogeneous Cellular System," in IEEE Network, vol. 31, no. 4, pp. 56-61, July 2017.

[5] A. Fotouhi, H. Qiang, M. Ding, M. Hassan, L. G. Giordano, A. GarciaRodriguez, and J. Yuan, "Survey on UAV Cellular Communications: Practical Aspects, Standardization Advancements, Regulation, and Security Challenges," in IEEE Communications Surveys Tutorials, pp. 1-1, 2019.

[6] M. Mozaffari, W. Saad, M. Bennis, Y. Nam, and M. Debbah, "A Tutorial on UAVs for Wireless Networks: Applications, Challenges, and Open Problems," in IEEE Communications Surveys Tutorials, pp. 1-1, 2019.

[7] A. AL-Hourani, S. Chandrasekharan, G. Kaandorp, W. Glenn, A. Jamalipour, and S. Kandeepan, "Coverage and rate analysis of aerial base stations," in IEEE Transactions on Aerospace and Electronic Systems, vol. 52, no. 6, pp. 3077-3081, December 2016.

[8] D. G. Cileo, N. Sharma, and M. Magarini, "Coverage, capacity and interference analysis for an aerial base station in different environments," in International Symposium on Wireless Communication Systems (ISWCS), Aug 2017, pp. 281-286.

[9] A. Al-Hourani, S. Kandeepan, and S. Lardner, "Optimal LAP Altitude for Maximum Coverage," in IEEE Wireless Communications Letters, vol. 3, no. 6, pp. 569-572, Dec 2014.

[10] L. Wang, B. Hu, and S. Chen, "Energy Efficient Placement of a Drone Base Station for Minimum Required Transmit Power," in IEEE Wireless Communications Letters, pp. 1-1, 2018.

[11] M. Alzenad, A. El-Keyi, F. Lagum, and H. Yanikomeroglu, "3-D Placement of an Unmanned Aerial Vehicle Base Station (UAV-BS) for Energy-Efficient Maximal Coverage," in IEEE Wireless Communications Letters, vol. 6, no. 4, pp. 434-437, Aug 2017.

[12] R. I. Bor-Yaliniz, A. El-Keyi, and H. Yanikomeroglu, "Efficient 3D placement of an aerial base station in next generation cellular networks," in IEEE International Conference on Communications (ICC), May 2016, pp. 1-5.

[13] A. V. Savkin and H. Huang, "Deployment of Unmanned Aerial Vehicle Base Stations for Optimal Quality of Coverage," in IEEE Wireless Communications Letters, vol. 8, no. 1, pp. 321-324, Feb 2019.

[14] X. Zhang and L. Duan, "Fast Deployment of UAV Networks for Optimal Wireless Coverage," in IEEE Transactions on Mobile Computing, vol. 18, no. 3, pp. 588-601, March 2019.

[15] M. Mozaffari, W. Saad, M. Bennis, and M. Debbah, "Efficient Deployment of Multiple Unmanned Aerial Vehicles for Optimal Wireless Coverage," in IEEE Communications Letters, vol. 20, no. 8, pp. 16471650, Aug 2016.

[16] H. Wu, X. Tao, N. Zhang, and X. Shen, "Cooperative UAV ClusterAssisted Terrestrial Cellular Networks for Ubiquitous Coverage," in IEEE Journal on Selected Areas in Communications, vol. 36, no. 9, pp. 2045-2058, Sep. 2018.

[17] B. Galkin, J. Kibilda, and L. A. DaSilva, "Coverage Analysis for Low-Altitude UAV Networks in Urban Environments," in IEEE Global Communications Conference, Dec 2017, pp. 1-6.

[18] H. Shiri, J. Park, and M. Bennis, "Massive Autonomous UAV Path Planning: A Neural Network Based Mean-Field Game Theoretic Approach," in arXiv e-prints, p. arXiv:1905.04152, May 2019.

[19] H. Kim, J. Park, M. Bennis, and S. Kim, "Massive UAV-to-Ground Communication and its Stable Movement Control: A Mean-Field Approach," in IEEE 19th International Workshop on Signal Processing Advances in Wireless Communications (SPAWC), June 2018, pp. 1-5.

[20] P. Series, "Propagation data and prediction methods required for the design of terrestrial broadband radio access systems operating in a frequency range from 3 to $60 \mathrm{GHz}$," Recommendation ITU-R, pp. $1410-1415,2013$. 